useful introduction to anyone concerned with elucidating the significance of particular variations of reaction rate with changes in the substrate or the reagent or the medium, for he will then stand a chance at least of being able to correlate his own observations with others which have been reported and which may be better understood. My view is that the chief value of linear free energy relation. ships is their use as a probe in understanding the mechanisms of reactions, the properties of solvents, and so on, and I am sorry that Wells did not give greater emphasis to this by the inclusion of more examples.

$$
\text { R. O. C. Norman }
$$

\section{HYDROCARBON CATALYSIS}

\section{Catalytic Conversion of Hydrocarbons}

By J. E. Germain. Pp. xi +322 . (Academic Press: London and New York, January 1969.) $80 s$; $\$ 12$.

DESPITE the profusion of fundamental studies of catalysts and catalytic reactions, most of the large-scale catalytic processes currently in use have been discovered chiefly by empirical methods. Nevertheless, Professor Germain believes that a stage has now been reached where "theoretical considerations are not completely useless, even for very practical chemists". In particular, he suggests that further important developments in the science of catalysis will emerge only if attempts are made to reconcile the chemical and physical approaches to the subject. Thus he points out in the preface that the most fully understood catalytic reactions are those in homogeneous systems where intermediate species have been identified by chemical means. The field of heterogeneous catalysis, in which physical factors also manifest themselves, however, demonstrates the shortcomings of the chemical approach on its own.

In view of the enormous volume of available material on the scientific and industrial aspects of catalysis in gencral, the author has limited his discussion to reactions of hydrocarbons either on their own or involving other simple species such as hydrogen, oxygen, water and carbon monoxide. The finding that hydrocarbons can be catalytically converted to other commercially important products gave great impetus to the study of such reactions in the period between the First and Second World Wars, and the field of hydrocarbon catalysis thus provides a very good testing ground for Germain's principal theses.

The author starts by examining the most likely mechanisms of interaction between hydrocarbons and catalysts of different types. After considering the structure of the intermediates involved in catalysis, which are either free radical or ionic species, he discusses the structure and reactions of labile inorganic complexes and then considers those catalytic reactions of hydrocarbons which are best explained in terms of the two types of intermediate.

The next two chapters deal with reactions involving the formation and rupture of $\mathrm{C}-\mathrm{H}$ bonds and $\mathrm{C}-\mathrm{C}$ bonds respectively. In the first, useful accounts are given of hydrogen-deuterium exchange and of hydrogenation, dehydrogenation and hydrogen transfer reactions. The chapter on $\mathrm{C}-\mathrm{C}$ bund reactions includes sections on isomerization, alkylation and hydrogen transfer reactions, and on the polymerization and cracking of various classes of hydrocarbons. The last two chapters of the book provide valuable accounts of the catalytic reactions of hydrocarbons with oxygen and carbon oxides respectively.

The author's approach to the subject is novel and refreshing inasmuch as he has brought into a logical and coherent pattern a large number of catalytic reactions of various types. The book certainly contains a mass of useful information and a detailed and up-to-date bibliography. It is perhaps difficult to digest all the facts and firsures on a first examination. but the book becomes more readable once it is realized that the main object is to illustrate certain broad trends and generalizations rather than to present the underlying details on which the conclusions are based. The style in which the book is written is perhaps a little awkward and stilted in places, but this does not appreciably detract from the great usefulness of a work which should be welcomed by all those interested in the general field of catalysis and in the reactions of hydrocarbons.

C. F. Cullis

\section{HETEROGENEOUS CATALYSIS}

Heterogeneous Catalysis

By S. J. Thomson and G. Webb. (University Chemical Texts.) Pp. $x+197$. (Oliver and Boyd: Edinburgh and London, February 1969.) $25 s$ paper; $42 s$ boards.

This latest addition to "University Chemical Texts" aims to give an up to date account in the space of some 200 pages of the rapidly expanding subject of heterogeneous catalysis. The authors inevitably have had to be highly selective in their choice of topics and depth of treatment: in this way they have been successful, bearing in mind that the book is intended primarily for undergraduates. The book is not so overloaded with facts. theories and references as to discourage the beginner, nor yet so devoid of depth and of practical applications as to be without stimulus for research workers and industrialists. It will therefore have a wide appeal.

Chapter one sets the scene with a discussion of the nature of catalysis in relation to adsorption and activation energy, and of the nature, preparation and uses of typical catalysts. Next, in the longest chapter in the book. chemisorption is treated in detail, leading to discussion of the nature of the surface bond and of experimental techniques for investigating surface species. Band theory. the Fermi level, the electronic factor in catalysis and photocatalysis are included in the chapter on oxide catalysts, and then follows a standard treatment of the kinetics of surface reactions with an instructive example of a kinetic study. Treatment in some depth of exchange reactions of hydrocarbons and of metal-catalysed hydrogenation of unsaturated hydrocarbons occupies the next two chapters, the most detailed and specialized of the whole book, and is followed by brief chapters on bifunctional catalysts, radioactive tracer methods and electrocatalysis. In the final chapter, the authors return to the topic of bonding at surfaces, and briefly discuss heterogeneous catalysis in relation to homogeneous catalysis, crystal field theory and the thermochemical approach to catalysis.

The book, based as it is on the authors' experience in teaching, rightly emphasizes principles illustrated by selected examples. One cannot complain about the examples chosen but, with advantage, the chapter on bifunctional catalysts could have been extended and retitled so as to include the synthesis of ammonia and the oxidation and ammoxidation of olefins. Obviously, not everything that one needs to know about heterogeneous catalysis is in this limited volume, but on the other hand everything that is in the book needs to be known. The book is well produced, very reasonably priced and fulfils a need long felt both by students and research workers.

DUNCAN TAYLOR

\section{SOLID STATE CHEMISTRY}

lonic Crystals, Lattice Defects and Nonstoichiometry By N. N. Greenwood. Pp. viii + 194. (Butterworth: London, January 1969.) $32 s$.

DuRing the past few years several books have been published in the field of solid state chemistry; from the 